\section{Y's and wherefores of our origin - speculating about human speciation}

\author{
The Speciation of Modern Homo sapiens \\ TJ Crow \\ Oxford University Press, New York. 2002; 265 pp. \\ $£ 29.50$, hardback. ISBN 0-19-726246-5x
}

Heredity (2003) 90, 421. doi:10.1038/sj.hdy.6800236

\section{Reviewed by L Somerville}

Tim Crow's introduction to this collection of papers includes the bold claim that '..it is arguable that scrutiny of the origin of our species casts speciation, and may be evolutionary theory as it now stands, in a critical light'. Unfortunately, this volume neither casts much new light on the question of the speciation of modern humans nor is there any 'value added' by reading the papers contained in the collection as a group. The striking lack of discussion evident between the contributors makes it very difficult to work out the extent to which any given paper in the first two sections of the volume on 'The Origin of the Species' and 'Language and the Evolution of the Brain' is supportive of the hypothesis presented in the third section 'The Search for a Critical Event', which is Crow's theory that the key genetic event in the speciation of Homo sapiens involved the mutation of a single gene on the $\mathrm{Y}$ chromosome in the Xq21.3/Yp1 1.2 region of homology.

Taken separately, the papers given in the first section include clear overviews by Stringer and Mellars on, respectively, the broadly accepted African origin of both modern human morphology and behaviour. Collard's detailed treatment of 'Grades and Transitions in Human Evolution' is much more interesting in what it says about early hominid evolution than in its contribution to the event considered here, since his conclusion is that, from the fossil record, Neanderthals and Modern Humans are of the same grade. Tattersall's piece on saltation adds little to the picture painted by Stringer and Mellars, but interprets the evidence for the emergence of modern humans as showing a saltational change.

The second section deals with the interesting and important question of the origin and evolution of language. As with the first section, there are some excellent reviews here, including the differences in neural control of vocalisation in human and nonhuman primates (Ploog). Three of the papers (Corballis, Steele and Cook) relate to lateralisation, but only Corballis's review clearly addresses the topic in a way which relates to Crow's hypothesis - and in fact he contests it. Bickerton gives some interesting speculations on the evolution of language from protolanguage. Since both Bickerton's and Steele's accounts are gradualistic, and range over a broader timescale than the last half-million years, it would be possible for the reader to conclude that these do not fully support Crow's inclusion of language and laterality as part of a recent speciation event.

The final section contains three papers, which are most likely to be of interest to readers of Heredity. All concern the $X$ and $Y$ chromosomes and, in particular, the evolutionary significance of areas of homology on them. This section also includes Crow's account of his hypothesis, but in terms which I found rather unconvincing. The specific, and potentially interesting suggestion that a candidate gene is that for protocadherin on the $Y$ chromosome is made, but then no further information is given about the known role of cadherins in brain development (Stt Redies, 2000). In neither Crow's paper nor the otherwise excellent review by Sargent et al is there any consideration of how changes in the cadherin genes on the sex chromosomes could lead to speciation, given the existence of other members of this gene family on the autosomes, although Sargent et al do give a very full account of what further tests should be carried out on the protocadherin genes PCDHX/PCDHY. Clearly, the sex chromosomes in the hominid line have undergone considerable change during the time since that lineage diverged from the great apes. The $\mathrm{Xq} / \mathrm{Yp}$ transposition itself appears to have occurred by 3 million years ago, i.e. closer to the origin of genus Homo than to the speciation of modern Homo sapiens.

Given that a key element in Crow's hypothesis is the role of sexual selection, it is strange that there is only one paper in the volume on this. Further, Rheinhold's contribution focuses on the reasons for X-linkage of sexually selected traits! It would have been stimulating to have a broader account of the possible role of sexual selection in human evolution (eg Miller, 2002).

This volume adds to the speculation around the origins of our own species. The paper by Sargent et al shows that such speculation can lead to testable hypotheses. The reader is left to do the work required to get any additional value out of these papers as a collection, although her reward is knowing where some clear overviews and excellent reviews can be found.

\section{References}

Miller G (2000). The Mating Mind. William Heinemann: Oxford. Redies C (2000). Cadherins in the central nervous system. Prog Neurobiol 61: 611-648

L Somerville School of Biological Sciences, University of Sussex Brighton, East Sussex BN1 9QG, UK, E-mail: lizsom@biols.susx.ac.uk 